University of Nebraska - Lincoln

DigitalCommons@University of Nebraska - Lincoln

1999

\title{
Effects of 4-n-Nonylphenol on Life-History Traits and Population Dynamics of a Polychaete
}

\author{
Flemming T. Hansen \\ Roskilde University \\ Valery E. Forbes \\ University of Nebraska-Lincoln, veforbes@umn.edu \\ Thomas L. Forbes \\ National Environmental Research Institute, Roskilde, Denmark
}

Follow this and additional works at: https://digitalcommons.unl.edu/biosciforbes

Part of the Pharmacology, Toxicology and Environmental Health Commons

Hansen, Flemming T.; Forbes, Valery E.; and Forbes, Thomas L., "Effects of 4-n-Nonylphenol on LifeHistory Traits and Population Dynamics of a Polychaete" (1999). Valery Forbes Publications. 8.

https://digitalcommons.unl.edu/biosciforbes/8

This Article is brought to you for free and open access by the Papers in the Biological Sciences at DigitalCommons@University of Nebraska - Lincoln. It has been accepted for inclusion in Valery Forbes Publications by an authorized administrator of DigitalCommons@University of Nebraska - Lincoln. 


\title{
EFFECTS OF 4-n-NONYLPHENOL ON LIFE-HISTORY TRAITS AND POPULATION DYNAMICS OF A POLYCHAETE
}

\author{
Flemming T. Hansen, ${ }^{1}$ Valery E. Forbes,${ }^{1,3}$ And Thomas L. Forbes ${ }^{2}$ \\ ${ }^{1}$ Department of Life Sciences and Chemistry, Roskilde University, PO Box 260, DK-4000 Roskilde, Denmark \\ ${ }^{2}$ Department of Marine Ecology and Microbiology, National Environmental Research Institute, P.O. Box 358, \\ Frederiksborgvej 399, DK-4000 Roskilde, Denmark
}

\begin{abstract}
A Life-Table-Response Experiment lasting $78 \mathrm{~d}$ was performed to investigate the toxic effects of sediment-associated 4-n-nonylphenol (NP) on growth, reproduction, and survivorship of isolated hermaphrodites of the infaunal polychaete Capitella sp. I. Demographic effects were evaluated using both a fully age-classified and a simple twostage model to estimate population growth rates $(\lambda)$. Decomposition analysis was performed to explore the contributions of each of the affected life-history traits to the effects observed on $\lambda$. Elasticity analysis was applied to examine the relative sensitivity of $\lambda$ to changes in each of the different life-history traits under different exposure levels.

In the lowest NP treatment (14 $\mu \mathrm{g} \mathrm{NP/g}$ dry mass of sediment) significant stimulatory effects were observed for both asymptotic body volume and average brood size, but these did not result in a significant effect on $\lambda$. Negative effects on brood size, volume-specific fecundity, time to first reproduction, and individual growth rate were significant in the highest NP treatment $(174 \mu \mathrm{g} / \mathrm{g}$ dry mass), and these effects resulted in a significant reduction in $\lambda$. Decomposition of the two-stage model indicated that the effect of NP on time to first reproduction was a major cause of changes in population growth rate. Although time to first reproduction increased by only $\sim 20 \%$ in the highest NP treatment relative to the control, it was responsible for more than half $(55 \%)$ of the effect on $\lambda$. In contrast, perindividual fecundity decreased by $75 \%$ but only explained $44 \%$ of the effect on $\lambda$. Elasticity analysis of the two-stage model showed that $\lambda$ became less sensitive to changes in fecundity and time to maturity, but not to changes in juvenile and adult survival probabilities, with increasing NP exposure.

In this study, population growth rate was not significantly affected by NP concentrations lower than those affecting the individual life-history traits. However, since the populationlevel consequences of changes in each trait depend on the starting value of $\lambda$, on the extent to which the other traits are impacted by the toxicant, and on the life-history characteristics of the species under consideration, the application of demographic analyses to chronic toxicity test results is required to link individual-level responses to population-level impacts of toxicant exposure.
\end{abstract}

Key words: alkylphenol ethoxylates; Capitella; demographic analysis; deposit feeder; hermaphrodites; pollution.

\section{INTRODUCTION}

The application of ecotoxicological tests of individual organism responses to toxicant exposure for ecological risk assessment requires that such responses be in some way predictive of effects occurring at higher levels of ecological organization. Toxicant effects on the life-history traits of individuals, such as survivorship, reproductive output, and maturation time, can be linked to population-level consequences by applying demographic models to calculate population growth

Manuscript received 3 November 1997; revised 19 March 1998; accepted 11 June 1998. For reprints of this Invited Feature, see footnote 1, page 429.

${ }^{3}$ Address correspondence to this author. E-mail: vforbes@virgil.ruc.dk rate $(\lambda)$. The extent to which population growth rate responds to toxicant exposure depends on the severity of the toxicant's effects on the individual life-history traits as well as on the sensitivity of $\lambda$ to changes in each of the individual traits contributing to it (Levin et al. 1996). In the demographics literature the term "sensitivity" is used to describe the strength of the relationship between $\lambda$ and the individual life-history traits contributing to it. To distinguish demographic sensitivity from toxicological sensitivity (i.e., the strength of the response of traits to toxicant exposure), we use the term "responsiveness" to refer to the latter. Thus a trait that is "responsive" has a low effect concentration (e.g., $\mathrm{EC}_{50}$ ).

The relationship between individual-level and population-level responses to toxicant exposure can take 
three forms, the consequences of which for risk assessment vary widely:

1) $\lambda$ is less responsive to toxicant exposure than the most toxicant-responsive life-history trait. This would be expected to occur when traits with low sensitivity (with respect to $\lambda$ ) are the most responsive to toxicant exposure, or when diverging/compensating effects among individual traits occur, e.g., when reproductive output is reduced and survivorship is increased significantly, resulting in an insignificant effect on $\lambda$. In this case, using the most toxicant-responsive trait(s) as measurement endpoint(s) in toxicity tests would yield a predicted no-effect concentration (PNEC) lower than that needed to prevent effects from occurring at the population level.

2) $\lambda$ is more responsive to toxicant exposure than the most toxicant-responsive life-history trait. This could occur when there are minor (i.e., not detectably significant) effects on more than one trait that combine to cause a significant reduction in $\lambda$. In this case using the most toxicant-responsive trait(s) as measurement endpoint(s) in toxicity tests could yield a PNEC that was not sufficiently low to protect against effects at the population level.

3) $\lambda$ is as responsive to toxicant exposure as the most toxicant-responsive life-history trait. In this case using the most toxicant-responsive trait(s) as measurement endpoint(s) would yield a PNEC that was just sufficient to ensure against effects occurring at the population level.

The extent to which each of these potential relationships applies to natural populations is unknown. But if individual-level ecotoxicological endpoints are to continue to be applied to predict ecological risks, it would be helpful to identify those situations for which ecological protection criteria are likely to be under- as well as over-protective. The undesirable costs associated with under-protective criteria are largely ecological (e.g., unexpected loss of species), whereas the costs associated with over-protective criteria are largely economic (e.g., excessive management costs) (Calow and Forbes 1999), and ideally both types of costs should be minimized.

This study was designed to: (1) investigate chronic effects on growth, fecundity, and survival in the polychaete Capitella sp. I exposed to 4-n-nonylphenol; (2) model the effects on population growth rates $(\lambda)$ of different exposure levels of 4-n-nonylphenol, and identify the life-history traits mainly responsible for the effects on $\lambda$; and (3) examine the sensitivity of $\lambda$ to changes in each of the life-history traits, and investigate whether these sensitivities varied as a function of contaminant exposure.

AlkylPhenol EthOxylates (APEO) represent the third largest group of surfactants in use worldwide (Nimrod and Benson 1996), and NonylPhenol EthOxylates (NPEO) comprise $>80 \%$ of the APEOs (Naylor 1995).
Nonylphenol (NP) is the main degradation product of NPEO, exhibits a high isomeric variability, and is considered to be a relatively stable metabolite (Giger et al. 1981, 1984). APEOs are highly toxic to aquatic organisms and show increasing toxicity with decreasing ethoxylate chain length (Nimrod and Benson 1996). The toxicity of nonylphenol to a variety of aquatic organisms has been examined (McLeese et al. 1980, 1981, Weinberger et al. 1987, Granmo et al. 1989, Comber et al. 1993), and LOEC values down to 6.7 $\mu \mathrm{g} / \mathrm{L}$ (for shrimp; Naylor 1995) have been reported. Marcomini et al. (1990) found concentrations of NP and NPEO (with one and two ethoxylate groups) ranging from $0.15-13.7 \mu \mathrm{g} / \mathrm{g}$ dry mass in marine sediments, and Ahel et al. (1990) reported similar concentrations in river sediments (i.e., 0.51-13.1 $\mu \mathrm{g} / \mathrm{g}$ dry mass).

The deposit-feeding polychaete Capitella sp. I has been described as the most opportunistic of several sibling species comprising the genus formerly known as Capitella capitata (Grassle and Grassle 1976, Grassle 1980). Capitella sp. I is common in areas with high organic input (Grassle and Grassle 1974, Forbes et al. 1994) where densities exceeding 200000 individuals/ $\mathrm{m}^{2}$ have been reported (Grassle and Grassle 1974, Sanders et al. 1980). Areas with high organic input are often associated with relatively high concentrations of contaminants (Gray 1979, Sanders et al. 1980, Ward and Hutchings 1996), and this, together with a feeding strategy that involves the consumption of large quantities of fine-grained sediment, means that Capitella sp. I populations are likely to be exposed to high concentrations of sediment-associated pollutants.

The life history of Capitella sp. I is complex and at least partly environmentally controlled. Female worms remain as functional females for their entire life-span, whereas a fraction of male worms develop into simultaneous hermaphrodites, which only rarely self-fertilize (Petraitis 1990)). The likelihood that a male worm develops into an hermaphrodite is negatively related to the presence of mature females in the population and to overall population density (Holbrook and Grassle 1984, Petraitis 1985, 1988, 1990), and almost $100 \%$ of isolated males turn into hermaphrodites (Petratitis 1990). This feature of Capitella's life history is likely to be of key importance to population dynamics (e.g., at low population density, under unfavorable conditions, or in the early stages of population recovery). For this reason we focus our analyses on the response of isolated hermaphrodites of Capitella sp. I exposed to 4-n-nonylphenol (NP).

\section{Materials And Methods Sediment properties and spiking}

Sediment for culturing worms and for use in the experiments was collected from the Isefjord (Zealand, Denmark) in October 1995, sieved $(<250 \mu \mathrm{m})$ and subsequently frozen $\left(-20^{\circ} \mathrm{C}\right)$. Organic content was deter- 
mined by loss of mass on ignition after combusting dried $\left(24 \mathrm{~h}\right.$ at $\left.80^{\circ} \mathrm{C}\right)$ sediment samples at $500^{\circ} \mathrm{C}$ for 6 h. $\mathrm{C}$ and $\mathrm{N}$ content were determined using a Carlo Erba model 1106 CHN microanalyzer (Carlo Erba, Milan, Italy) following Hedges and Stern (1984). Organic content of the sediment after sieving (mean $\pm 1 \mathrm{SD}$ ) was $7.4 \pm 0.2 \%$. The $\mathrm{C}$ and $\mathrm{N}$ contents were $3.85 \pm 0.11 \%$ and $0.41 \pm 0.02 \%$, respectively.

The nominal sediment concentrations selected for spiking were 0 (NP0), 20 (NP1), 100 (NP2), and 185 (NP3) $\mu \mathrm{g} \mathrm{NP/g}$ dry mass, to cover the range from environmentally realistic concentrations to those approaching acutely toxic effect concentrations for growth of Capitella sp. I determined in a pilot study (F. Hansen, unpublished data). NP was dissolved in a minimum of acetone $(\sim 1 \mathrm{~mL})$. Spiking was performed by adding the 4-n-nonylphenol-acetone solution to 2 $\mathrm{kg}$ of wet sediment in a 4-L flask which was shaken/ rotated for $24 \mathrm{~h}$ in darkness. To enhance solubility and to ensure uniform mixing, all flasks were heated to $50^{\circ} \mathrm{C}$ and reshaken for $5 \mathrm{~h}$. The spiked sediment was blended for $15 \mathrm{~s}$ before being divided into smaller portions and frozen $\left(-20^{\circ} \mathrm{C}\right)$. Control sediment was treated as spiked sediment including the addition of acetone.

\section{Concentration measurements}

To measure the actual concentration of NP in treated sediments, we used a modified procedure for sediment extraction of organochlorines (Jensen et al. 1977) and measured silylated derivatives of NP on a gas-chromatograph (GC) (Hewlett-Packard, Model 5890 Series II; HP-5 MS, Crosslinked 5\% Ph Me silicone column) coupled with a mass spectrometer (MS) (Hewlett-Packard, Model 5971A, Birkerød, Denmark). The amount of NP in the sediment samples was measured relative to an internal standard (4-octylphenol) added prior to extraction. Extraction procedures as described by Jensen et al. (1977) have been used for measurements of 4-nonylphenol in sewage sludge (Wahlberg et al. 1990), and we adjusted this protocol as follows.

Sediment samples were freeze dried, and a sample of $0.5-1.0 \mathrm{~g}$ of sediment was added to a glass centrifuge tube with a teflon-sealed screw cap. An amount of internal standard was added equivalent to the amount of $\mathrm{NP}$ expected in the sediment sample. Then $2 \mathrm{~mL}$ of $5.5 \mathrm{~mol} / \mathrm{L}$ acetic acid was added to attain a $\mathrm{pH}$-value of 1-2. This was done to avoid possible adsorption of the phenolic compounds on alkaline sites due to a relatively high concentration of calcium carbonates in the sediment (T. Lund, personal communication). Earlier attempts without addition of acid showed unsatisfactory recovery percentages. After $30 \mathrm{~min}$ the glass tubes were centrifuged at $5.6 \times 10^{3} \mathrm{~m} / \mathrm{s}^{2}$ for $10 \mathrm{~min}$, and the supernatant was removed. Three milliliters of acetone were added, and the tubes were shaken immediately and treated with ultrasound for $10 \mathrm{~min}$ to disrupt coagulating particles. The centrifuge tubes were shaken thoroughly on a vortex shaking table for $30 \mathrm{~min}$, and thereafter centrifuged for an additional $10 \mathrm{~min}$. The supernatant was transferred with a pasteur pipette, through water-resistant cotton, to a rotation flask. The extraction procedure was repeated twice, the second time with a mixture of acetone $(1 \mathrm{~mL})$ and $\mathrm{n}$-hexane (3 mL).

The extracts were evaporated to total dryness using a rotation evaporator. Acetonitrile $(2-3 \mathrm{~mL})$ and 100 $\mu \mathrm{L}$ of BSA (N,O-bis-(trimethylsilyl)-acetamide) were added, and the glassware was placed at $50^{\circ} \mathrm{C}$ for $1 \mathrm{~h}$ to obtain silylated derivatives of 4-n-nonylphenol. This was necessary to ensure reproducible results using the column available in the GC-MS. This step can be ignored using more appropriate columns, see Sweetman (1993).

HPLC-grade acetonitrile and n-hexane were obtained from Niels Peter Mark, Valby, Denmark. BSA (N,OBis-(trimethylsilyl)acetamide), 4-octylphenol and 4-(tertoctyl)phenol, acetone, and acetic acid (both pro-analytic) were obtained from Bie and Berntsen, Rødovre, Denmark. 4-n-nonylphenol was obtained from Lancaster Synthesis Limited, Eastgate, UK. All experimental items were cleaned in $10 \%$ nitric acid solution for a minimum of $24 \mathrm{~h}$ before use.

The recovery percentage of NP (mean $\pm 1 \mathrm{SD}$ ) was $42.5 \pm 8.0 \%$. (Reported values have been corrected for percent recovery.) The detection limit was $\sim 0.1 \mu \mathrm{g} / \mathrm{g}$ dry mass.

\section{Experimental design and worm measurements}

Worms were originally obtained from Setauket Harbor, New York, USA, in 1984, and identified as Capitella sp. I by J. P. Grassle. Laboratory stock cultures of Capitella sp. I were reared at $20^{\circ} \mathrm{C}$ in aerated aquaria containing $27 \%$ o North Sea seawater and sediment (described above). To maintain worm cultures, but not for NP experiments, ground fish food (Tetra Min, Tetra/ Second Nature, Blacksburg, Virginia) was added regularly as a supplementary food source.

Four brood tubes with newly released larvae were collected from laboratory cultures. Larvae from each brood were picked randomly and distributed evenly among 12 glass beakers $(250 \mathrm{~mL}$ diameter $=6.8 \mathrm{~cm})$. There were three glass beakers for each of the three treatment concentrations and the control. To each beaker were added 33 larvae (i.e., 99 larvae per treatment; giving a density of 9090 worms $/ \mathrm{m}^{2}$ ). Each glass beaker was filled with filtered seawater $(<0.2 \mu \mathrm{m})$ and a thin layer of contaminated sediment $(\sim 3 \mathrm{~mm})$. Sediment was defrosted and blended for $10 \mathrm{~s}$ before addition to vials or beakers to ensure uniform NP-concentrations between replicates and to disrupt any clumps of sediment that had formed during freezing. Since larval settlement occurs within hours of release in the presence of sediment (Pechenik and Cerulli 1991), day zero was defined as the day larvae were added to the beakers. 
A
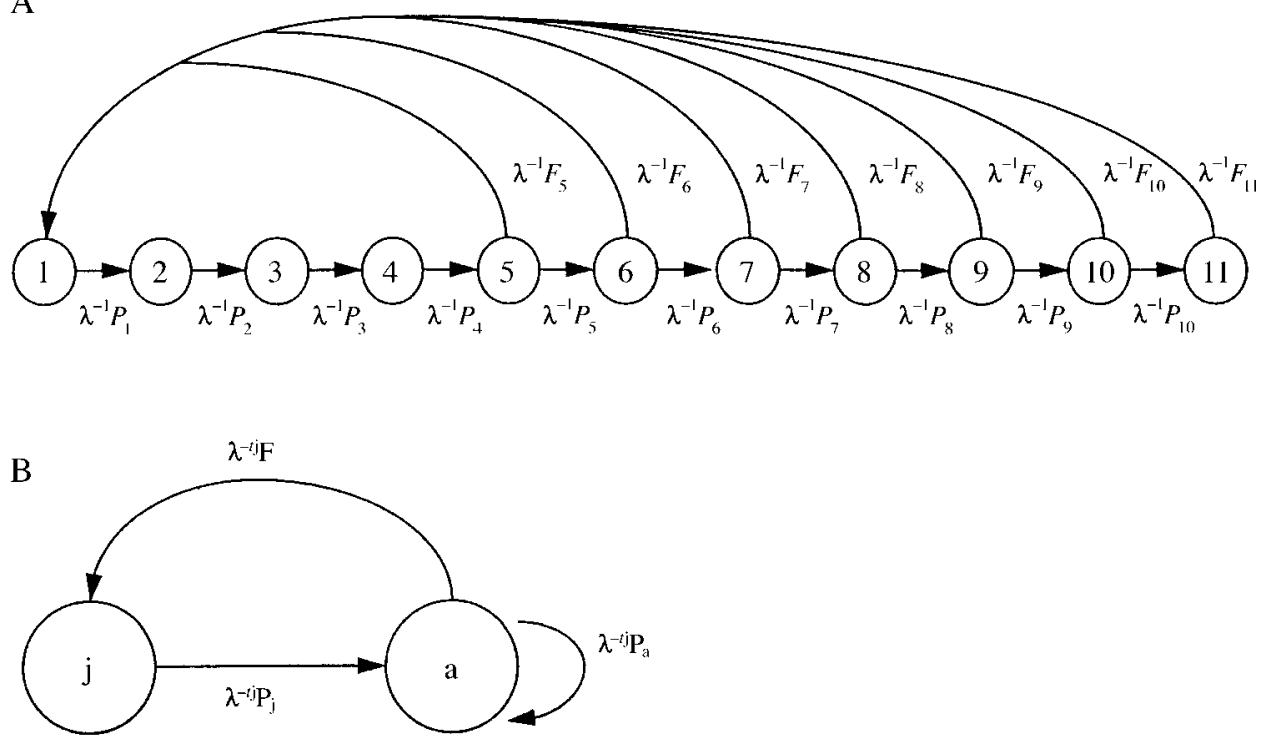

FIG. 1. Life-cycle graphs representing the two population models used for demographic analysis. (A) The age-classified model with 11 age classes each of 1 -wk duration. $P$ values denote the survival probabilities from one age class to the next, indicated by arrows. $F$ values denote number of offspring (age class 1 ) per individual produced by the corresponding age class $i$; $\lambda$ is the population growth rate. (B) The two-stage model with a juvenile (j) and an adult (a) stage. $P_{\mathrm{j}}$ denotes survival probability from newly hatched larvae to maturity, $P_{\mathrm{a}}$ denotes adult survival probability, $F$ denotes adult per individual fecundity, and $t_{\mathrm{j}}$ represents time to first reproduction. (See Materials and methods for further explanation.)

All beakers were covered with parafilm to reduce evaporation, aerated continuously, and maintained in darkness (to reduce NP degradation). No supplemental food was added to the experimental sediment. Salinity and temperature were monitored at least every 2 nd or 3 rd day during the experiment and were $20^{\circ} \mathrm{C}$ and $26-27$ $\%$, respectively. To maintain constant treatment levels during the experiment, worms were placed in new sediment and seawater approximately every week (i.e., on days $7,14,21,25,32,39,46,53,60,66$, and 72), and samples of sediment were taken before and after transfer for analysis of 4-n-nonylphenol (NP).

Survival and worm body volume were measured the first time on day 14 and then each time worms were transferred to new sediment (approximately once every week). Body sizes were measured using a video camera mounted on a stereo microscope. Body length $(L)$ and area $(A)$ were estimated with the JAVA program (Jandel Incorporated, Ekrath, Germany), and body volumes (BV) were calculated assuming a cylindrical body form $\left(\mathrm{BV}=\left[\left(\pi \times A^{2}\right) /(4 \times L)\right]\right.$; Forbes and Lopez 1987). All measurements of body length and area were the means of triplicate determinations $(1 \mathrm{SD}<10 \%)$. Until day 25 eight worms were picked randomly from each of the 12 glass beakers and their body volumes measured. After day 25, repeated measurements of the same individuals (20 per treatment) were made on each census day. On day 25 all individuals were sexed. Twenty males were picked randomly from each concentration and transferred, individually, to $20-\mathrm{mL}$ vials (diameter $=2.5 \mathrm{~cm}$ ) containing $6-8 \mathrm{~mL}$ of sediment. Life-table parameters were obtained from these isolated males, all of which developed into hermaphrodites. The 20 vials for each concentration were placed in a $1.5-\mathrm{L}$ crystallizing dish filled with filtered seawater, which was continuously aerated and covered to avoid evaporation.

As soon as ovaries appeared in an isolated male it was mated with a mature $\left(>2 \mathrm{~mm}^{3}\right)$ male from the stock culture, and the pair was kept together for the remainder of the experiment (density $=4074$ worms $/ \mathrm{m}^{2}$ ). Mating males that later developed ovaries were replaced by a new mature male. Each time a brood was laid, the adult worm was gently separated from the brood tube, and the broods were preserved in $4 \%$ formaldehyde until counting. Bridges et al. (1994) found that premature removal of young did not affect per brood fecundity or brooding frequency in Capitella sp. I. Despite careful handling of the animals, a few individuals were damaged severely and were discarded. Data from these individuals were not included in the final data set. The experiment was terminated on day 78 when the number of broods produced was sufficient for determining $\lambda$ (Levin et al. 1996).

\section{Demographic analysis}

Demographic analysis and calculation of population growth rates were carried out using both age-classified (with 1-wk intervals) and two-stage models following Levin et al. (1996). With the age-classified model (Fig. 1A) it is possible to determine the most important age classes influencing population growth rate. The age- 
classified model, however, does not include time to first reproduction as an explicit parameter. To incorporate time to first reproduction in the demographic analysis, a simple two-stage model was employed (Fig. 1B; Caswell 1996, Levin et al. 1996). The parameterization of the two-stage model is partly based on the age-classified model as described below.

The equations and computation procedures used in the present study have been described thoroughly in Levin et al. (1996) and Caswell (1989a, b, 1996) and will only be described briefly here. All computations were performed using MACSYMA Ver. 2.1 (Arlington, Massachusetts).

\section{The age-classified model}

Life-table experiments provide data on the probability of survival from birth to age $x\left(l_{x}\right)$, and the number of offspring per individual aged $x$ per unit time $\left(m_{x}\right)$. The age-classified model was constructed as a population projection matrix (Leslie 1945). When parameterizing the population projection matrix with survivorship and fecundity terms, $l_{x}$ and $m_{x}$ have to be transformed to corresponding $P_{i}$ and $F_{i}$ values, representing the entries in the population matrix. $F_{i}$ represents the average per individual fecundity in age class $i$, and $P_{i}$ denotes the probability of surviving from age class $i$ to age class $i+1$. Thus, $P_{i}$ and $F_{i}$ represent survival and fecundity terms for discrete age classes $i$, while $x$ in $m_{x}$ and $l_{x}$ represents a continuous age variable. $P_{i}$ and $F_{i}$ were calculated using the birth-flow formula outlined in Caswell (1989a) and given by Eqs. 1 and 2 in Levin et al. (1996).

Matrices were constructed with age intervals of 1 wk. However, data from the 78-d life table experiment were not obtained precisely every seventh day (see Experimental design and worm measurements). Therefore, prior to calculation of matrix entries, values for $l_{x}$ and $m_{x}$ corresponding to 1 -wk intervals were estimated using linear interpolation between data from each census day. Furthermore, data obtained for day 66 were estimated from linear interpolation between values from day 60 and day 72, instead of using actual values from the experiment. This was done because data from day 66 were influenced by a power failure for $2 \mathrm{~d}$ that caused unfavorable oxygen conditions, which reduced the number of breeding individuals observed on this day in all treatments. Fecundity $\left(F_{i}\right)$ was assigned to the week that larvae were released, assuming a 7 -d brood development period. Brood development of female offspring at $20^{\circ} \mathrm{C}$ is $6-8 \mathrm{~d}$ (Levin et al. 1996).

Population growth rates were estimated by solving the characteristic equation of each matrix for each treatment. The largest positive root $(\lambda)$ corresponds to the dominant eigenvalue of the matrix and expresses the rate at which a given population size increases over one time unit (i.e., $1 \mathrm{wk}$ ) at the stable age distribution. $\lambda$ is equal to $e^{r}$, where $r$ is the intrinsic rate of increase in a population as described by the Euler-Lotka equation (Caswell 1989a). When $\lambda=1$, the population is at steady state; if $\lambda<1$, the population is declining; if $\lambda>1$, the population is increasing.

Sensitivities of $\lambda$ to changes in the life-history traits $P_{i}$ and $F_{i}$ were calculated as the partial derivatives of $\lambda$, following Levin et al. (1996: Eqs. 3 and 4). In addition, the effect of each treatment on $\lambda$, measured relative to the control, was decomposed into contributions from each of the age-specific survivorship and fecundity terms following Caswell (1989b) and Levin et al. (1996: Eq. 5). In this way it was possible to identify the life-history traits that were most responsible for the effects of NP treatment on $\lambda$.

\section{The two-stage model}

The two-stage model (Fig. 1b) simplifies the population dynamics to four parameters, juvenile and adult survival probability $\left(P_{\mathrm{j}}\right.$ and $\left.P_{\mathrm{a}}\right)$, overall adult fecundity $(F)$, and time to first reproduction $\left(t_{\mathrm{j}}\right)$. Time to first reproduction is defined as $t_{\mathrm{j}}=m-1$, where $m$ is calculated as the first age class with a nonzero fecundity in the age-classified population matrix. $P_{\mathrm{j}}$ is simply the probability of surviving to age class $m$ and can be determined from the juvenile survival probability per unit time, $\sigma_{1}$, as $P_{\mathrm{j}}=\sigma_{1}^{t_{\mathrm{j}}}$. Adults survive with probability $P_{\mathrm{a}}=\sigma_{2} . P_{\mathrm{a}}$ and $F$ are calculated as weighted means of $P_{i}$ and $F_{i}$ in the reproductive age classes of the ageclassified matrix model, weighted with respect to the stable age distribution of the age-classified matrix model (Levin et al. 1996: Eqs 8 and 9). Population growth rate $(\lambda)$ is calculated as the largest positive root of the characteristic polynomial of the life-cycle graph describing the two-stage model in Fig. 1B (for details see Caswell 1989a). The sensitivity expressions of $\lambda$ to changes in each of the four life-history traits $\sigma_{1}, \sigma_{2}, F$, and $t_{\mathrm{j}}$, as well as decomposition of contributions from each of the four traits to treatment effects on $\lambda$, were calculated as in Levin et al. (1996: Eqs. 12-16). As for the age-classified model, sensitivities were evaluated for the two-stage model parameterized with the mean values of the parameters in the control and treatment models.

\section{Elasticity analysis}

To investigate the extent to which demographic properties changed with exposure level, sensitivity analyses were performed using the two-stage model for each treatment and the control as described above. Sensitivities represent the relative change in $\lambda$ with respect to changes in each of the life-history traits. For instance, if each life-history trait in turn is reduced by $10 \%$, this does not necessarily result in a $10 \%$ decrease in $\lambda$ in each case. The effect on $\lambda$ will vary depending on the sensitivity of $\lambda$ to changes in each of the parameters, and these sensitivities are determined by the 
life history of the organism as described by $F, P_{\mathrm{a}}, P_{\mathrm{j}}$, and $t_{\mathrm{j}}$. However, since survivorship, fecundity, and time to first reproduction are measured on different scales, absolute values of sensitivities cannot readily be compared among different parameters. To overcome this difficulty, it is possible to calculate a measure of relative sensitivity, known as "elasticity," $e_{\mathrm{a}}$ :

$$
e_{\mathrm{a}}=(a / \lambda)(d \lambda / d a)
$$

where $a$ denotes the life-history trait, and $d \lambda / d a$ is the sensitivity of $\lambda$ to changes in the life-history trait, $a$ (Caswell et al. 1984, de Kroon et al. 1986). Elasticity makes it possible to compare sensitivities of $\sigma_{1}, \sigma_{2}, F$, and $t_{\mathrm{j}}$ on a common scale.

\section{Statistics}

Brood size and volume-specific fecundity were analyzed using ANCOVA with time as the covariate. Since slopes for brood size vs. time did not differ from zero, one-way analysis of variance was chosen to test differences among treatments. However, Levene's test showed significantly heterogeneous variances, which could not be made homogeneous by transformation. Thus, the pairwise comparisons of brood size between treatments and the control were performed using Kruskal-Wallis nonparametric tests (Siegel and Castellan 1988). To perform pairwise comparisons on volumespecific fecundity between treatments and the control, a Dunnett's test was performed on elevations of the regression lines (Zar 1974).

Treatment effects on $t_{\mathrm{j}}$, number of broods per individual and size at first reproduction, were analyzed using one-way ANOVA followed by Dunnett's pairwise comparisons test when ANOVA results revealed significant differences among treatments. The KruskalWallis test was used to test treatment effects on time between broods, number of eggs per reproducing individual, and number of eggs per individual (includes reproducing and nonreproducing hermaphrodites), since no transformations were found to improve the homogeneity of variances sufficiently.

Both logistic and Gompertz growth models (Kaufmann 1981) were fitted to the sigmoid-shaped body volume vs. time curves, separately for each NP treatment, with the Gompertz model giving the better fit. The equations were estimated using the NONLIN-module in SYSTAT, which provided $95 \%$ confidence intervals on each equation parameter. Non-overlapping confidence intervals were defined as significantly different.

Differences in survivorship among treatments were tested by analyzing numbers of survivors in triplicates on day 25 for each treatment, using one-way ANOVA and Dunnett's pairwise comparisons test.

To test for significant differences among population growth rates, $95 \%$ confidence intervals were estimated for the age-classified model using the bootstrap pro- cedure recommended by Meyer et al. (1986). Nonoverlapping confidence intervals were defined as significantly different. Mortality data from the first $25 \mathrm{~d}$ were obtained from triplicates, whereas data on mortality, as well as fecundity, after day 25 were obtained from 18-20 replicates. Therefore, each bootstrap estimate was based on a combination of 18-20 random subsamples from triplicates representing early mortality until day 25 , and from the 18-20 replicates on fecundity and adult mortality. All bootstrap estimates of $\lambda$ were based on 1000 subsamples drawn from the original data set. Since the distributions of bootstrap estimates were very close to normal, confidence intervals were calculated by multiplying the bootstrap standard error by the critical $t$ value at the degrees of freedom corresponding to the sample size of the original data set, in this case the number of individuals followed from day 25. All bootstrap estimates were bias adjusted following Meyer et al. (1986).

Unless otherwise noted, numbers in parentheses are standard deviations. For all tests, $P<0.05$ was defined as significant. All statistical calculations were performed using SYSTAT (version 5.0).

\section{RESULTS}

\section{Sediment analysis}

Average concentrations of NP (mean $\pm 1 \mathrm{SD}$ ) measured in the experimental sediments just after addition were NP0, <0.1 (i.e., <detection limit); NP1, $14 \pm 7$ $\mu \mathrm{g} / \mathrm{g}$ dry mass; NP2, $52 \pm 3 \mu \mathrm{g} / \mathrm{g}$ dry mass; NP3, 174 $\pm 16 \mu \mathrm{g} / \mathrm{g}$ dry mass. During one week of exposure, sediment concentrations in NP1 and NP2 treatments decreased by about half, equivalent to a degradation rate of $\sim 10 \%$ per day. At the highest NP treatment no reduction in NP concentration was detected.

\section{Survivorship}

By day 25 nearly all individuals in the control (NP0) and the two lowest treatment concentrations (NP1 and NP2) had reached maturity (determined by the presence of ovaries or male copulatory setae) and consisted almost entirely of individuals with male characteristics. In the highest treatment concentration (NP3) no mature females were observed, and individuals without setae were very small and regarded as immature.

Juvenile survivorship was generally high $(>85 \%)$, and no significant differences were observed among treatments (ANOVA: $P=0.501$, df $=3,8, F=0.858$ ). No mortality was observed after day 25 except for one individual registered as dead on day 72 (in NP2).

\section{Body volume}

Gompertz equations for body volume measurements from day 14 to day 78 were characterized by three parameters: A, the asymptotic body volume; $\mathrm{B}$, the rate at which body volume approached asymptotic body volume; $\mathrm{C}$, a measure of the displacement of the Gom- 
TABLE 1. Estimated parameters of the Gompertz equations (BV $=A \times \exp [-\exp (-B \times(t-C))]$ ) fitted to body volume data for Capitella sp. I for each 4-n-nonylphenol treatment and the control, and corresponding $95 \%$ confidence intervals.

\begin{tabular}{|c|c|c|c|c|c|}
\hline \multirow[b]{2}{*}{ Concentration } & \multirow[b]{2}{*}{ Parameter } & \multirow[b]{2}{*}{ Estimate } & \multirow{2}{*}{$\begin{array}{l}\text { Adjusted } \\
\text { SE }\end{array}$} & \multicolumn{2}{|c|}{$95 \% \mathrm{CI}$} \\
\hline & & & & Low & High \\
\hline NP0 & A & 17.53 & 0.69 & 16.17 & 18.89 \\
\hline NP1 & A & $21.03 \dagger$ & 0.57 & 19.90 & 22.16 \\
\hline NP2 & A & 18.94 & 1.18 & 16.61 & 21.27 \\
\hline NP3 & A & 23.92 & 3.28 & 17.44 & 30.39 \\
\hline NP0 & B & 0.100 & 0.018 & 0.064 & 0.136 \\
\hline NP1 & B & 0.114 & 0.016 & 0.082 & 0.145 \\
\hline NP2 & B & 0.074 & 0.015 & 0.045 & 0.103 \\
\hline NP3 & B & $0.036 \dagger$ & 0.006 & 0.023 & 0.048 \\
\hline NP0 & $\mathrm{C}$ & 26.62 & 1.187 & -28.96 & -24.28 \\
\hline NP1 & $\mathrm{C}$ & 25.27 & 0.814 & -26.88 & -23.67 \\
\hline NP2 & $\mathrm{C}$ & 30.89 & 1.642 & -34.13 & -27.66 \\
\hline NP3 & $\mathrm{C}$ & $44.55 \dagger$ & 4.712 & -53.83 & -35.26 \\
\hline
\end{tabular}

Note: $\mathrm{A}=$ asymptotic body volume (cubic millimeters), $\mathrm{B}=$ the rate with which body volume approaches asymptotic body volume (per day), and $\mathrm{C}=$ horizontal displacement of the Gompertz curve to the right (days).

$\dagger$ Indicates when the difference from the control is significant due to nonoverlapping confidence intervals.

pertz curve to the right along the $x$ axis (Table 1). Worms in NP1 reached a larger asymptotic body volume than control worms. There was no difference in asymptotic body volume between NP0 and the two highest NP concentrations. Worms in NP3 approached their asymptotic body volume more slowly and had growth curves shifted significantly to the right compared to control worms (Table 1). While growth trajectories of NP0, NP1, and NP2 all reached an asymptote prior to the end of the experiment, this was not the case for NP3 (Fig. 2).

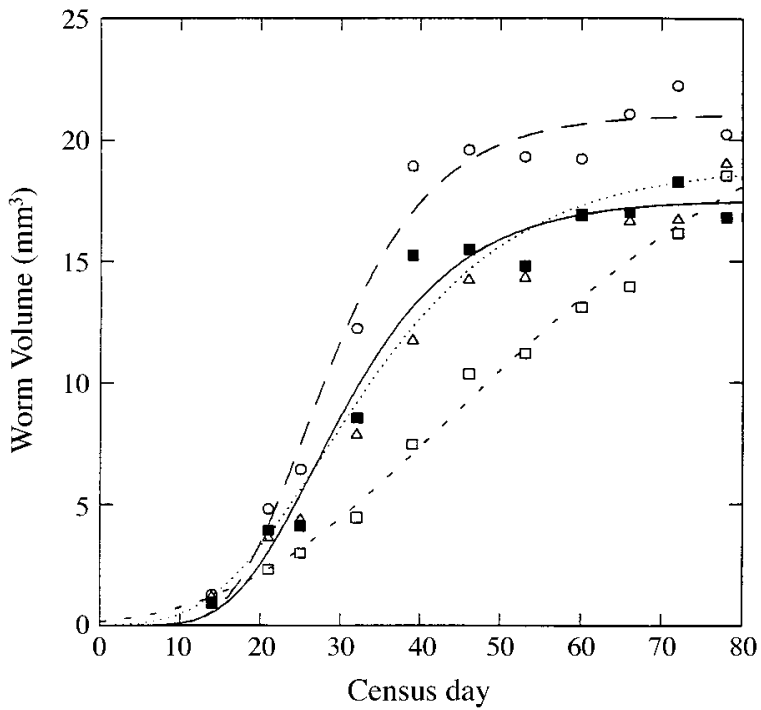

FIG. 2. Gompertz curves fitted to age-specific worm body volume measurements for each of the three 4-n-nonylpheno concentrations and the control. Each point represents a mean value for the day and treatment. Solid squares with a solid line fit, NP0; open circles with long-dash line fit, NP1; open triangles with dotted line fit, NP2; open squares with shortdash line fit, NP3. Corresponding parameter estimates are given in Table 1.

\section{Reproductive output}

Effects of NP on the number of eggs per brood showed similar patterns to the growth trajectories (Fig. $3)$. Treatment effects on average brood size were significant (ANCOVA, $P<0.001$, df $=3,70, F=62.65$ ), and time had no effect $(P=0.09$, df $=1,70, F=$ $2.92)$. Brood sizes were significantly larger $(P<0.001)$ in the NP1 treatment compared with NP0 and significantly smaller $(P<0.001)$ in the NP3 treatment compared with NP0. No difference was detected between NP2 and NP0.

Both time (ANCOVA, $P<0.001$, df $=1,70, F=$ 65.7) and treatment $(P<0.001, \mathrm{df}=3,70, F=45.2)$ had highly significant effects on volume-specific fecundity (Fig. 4). However, volume-specific fecundity of worms exposed to NP1 did not differ significantly from the control $(P>0.05)$, indicating that the larger brood sizes of NP1, relative to the control, were due primarily to the greater body size attained by NP1

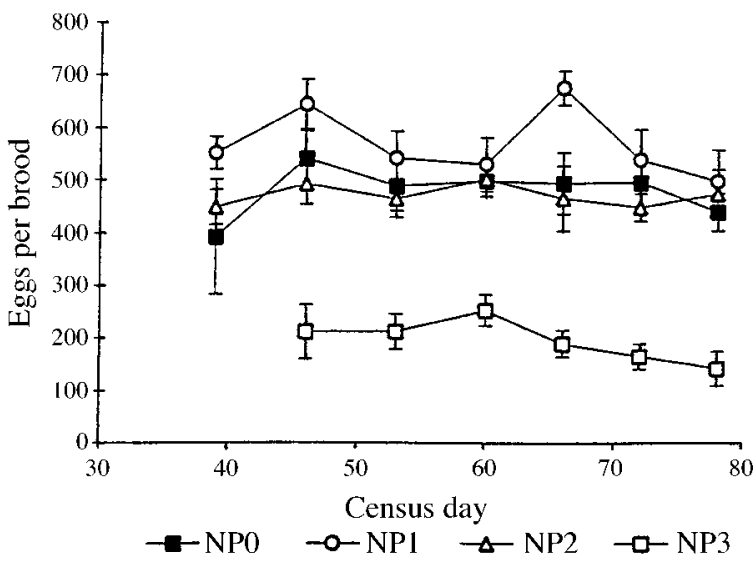

FIG. 3. Mean brood sizes (eggs per brood) for broods collected at each census day. Points indicate mean values. Error bars represent $\pm 1 \mathrm{SE}$. 


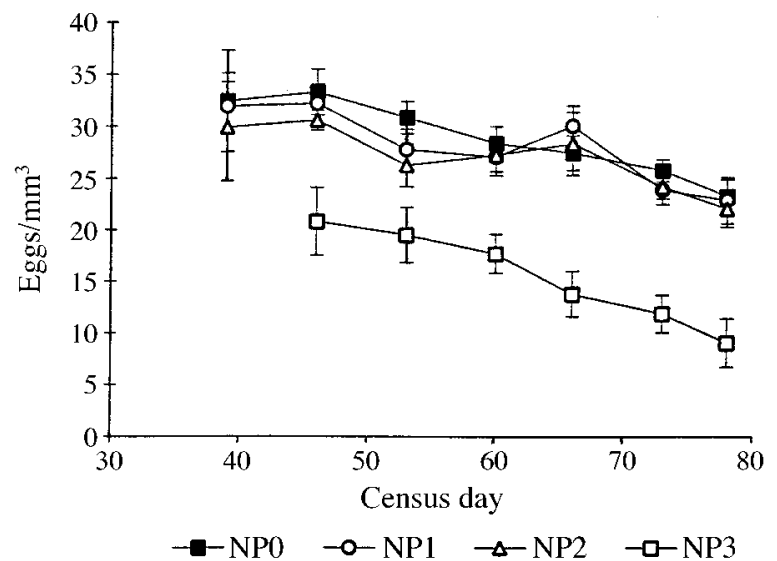

FIG. 4. Volume-specific fecundity for breeding individuals each census day. Points indicate mean values; error bars represent standard errors.

worms. Volume-specific fecundity for NP3 worms (but not NP2 worms) was significantly lower than that of control worms $(P<0.01)$.

The body volume of worms at the time of first reproduction was significantly reduced in NP3 $(P=$ 0.004 , df $=3,71, F=7.8$ ) relative to the control. No significant differences were observed between NP0, NP1, and NP2. Time to first reproduction (Table 2) was almost identical for NP0, NP1, and NP2, whereas the NP3 worms were delayed by $9-10 \mathrm{~d}$ relative to NP0 $(P<0.001, \mathrm{df}=3,71, F=15.7)$. Time between broods $\left(t_{\mathrm{a}}\right)$ was $48 \%$ longer in the NP3 treatment compared to the control $(0.01<P<0.05$, Kruskal-Wallis $=6.78)$.

\section{Population growth rates}

The population growth rates calculated by the ageclassified model (Fig. 5) are bias-adjusted bootstrap estimates \pm their $95 \%$ confidence limits. Although the

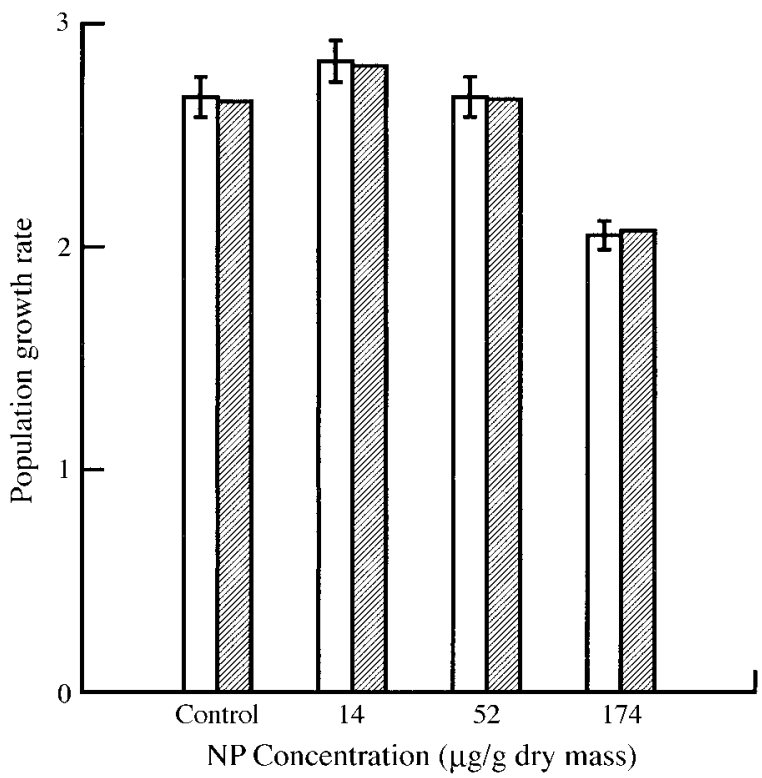

FIG. 5. Population growth rates, $\lambda$, for both the age-classified and two-stage models. Error bars represent $95 \%$ confidence intervals based on bootstrap replicates. Shaded bars, stage-classified model; open bars, age-classified model.

population growth rates estimated by the two-stage model were not bias adjusted, the two models yielded nearly identical results.

The positive response observed both in brood size and asymptotic body volume at the lowest NP treatment resulted in a $6 \%$ increase in $\lambda$, which was not significantly different from $\lambda$ estimated for control worms. The joint effect of changes in fecundity, time to first reproduction, and breeding frequency in the highest NP treatment, however, resulted in a significant $24 \%$ decrease in $\lambda$ relative to the control.

TABLE 2. Reproductive characteristics for individuals that bred at least once during the experimental period.

\begin{tabular}{lcccc}
\hline \hline \multirow{2}{*}{\multicolumn{1}{c}{ Characteristic }} & \multicolumn{3}{c}{ Concentration } \\
\cline { 2 - 5 } & \multicolumn{1}{c}{ NP0 } & NP1 & NP2 & NP3 \\
\hline No. of individuals $\dagger$ & 19 & 18 & 18 & 20 \\
No. of reproductive individuals $\neq$ & 17 & 18 & 16 & 20 \\
$t_{\mathrm{j}}$ (days) & $49.7( \pm 4.2)$ & $49.0( \pm 4.7)$ & $49.7( \pm 4.7)$ & $58.1 * * *( \pm 6.8)$ \\
$t_{\mathrm{a}}$ (days) & $7.3( \pm 2.0)$ & $7.8( \pm 2.4)$ & $7.5( \pm 2.5)$ & $10.69 *( \pm 4.9)$ \\
$t_{\mathrm{a}}$ (day 66) & $6.8( \pm 1.3)$ & $6.6( \pm 1.1)$ & $6.7( \pm 1.8)$ & $8.5( \pm 3.7)$ \\
No. broods per reproductive individual & $5.2( \pm 0.9)$ & $5.1( \pm 1.1)$ & $5.3( \pm 1.1)$ & $2.9 * * *( \pm 0.8)$ \\
No. eggs per reproductive individual & $2564( \pm 854)$ & $2876( \pm 1394)$ & $2490( \pm 1042)$ & $557 * *( \pm 311)$ \\
No. eggs per individual $\dagger$ & $2292( \pm 1140)$ & $2876( \pm 1394)$ & $2214( \pm 1041)$ & $557 * *( \pm 312)$ \\
Size at maturity $\left(\mathrm{mm}^{3}\right)$ & $16.9( \pm 5.7)$ & $18.8( \pm 6.4)$ & $14.2( \pm 3.7)$ & $11.4 * *( \pm 4.0)$ \\
Total no. broods & 89 & 92 & 84 & 58 \\
Total no. eggs & 43551 & 51764 & 39851 & 11140
\end{tabular}

Note: Time to first reproduction, $t_{\mathrm{j}}$, is calculated as the time from day 0 (i.e., larval settlement) to the day when eggs were produced (the mean between the first census day when eggs were recorded and the previous census day); $t_{\mathrm{a}}$ represents time between reproductive events both including and excluding day 66. Calculation of $t_{\mathrm{a}}$ is based on time between two reproductive events. Data are presented as means $( \pm 1 \mathrm{SD})$.

$* P<0.05, * * P<0.01, * * * P<0.001$.

$\dagger$ Includes all individuals.

$\ddagger$ Only includes individuals that bred at least once during the experiment. 


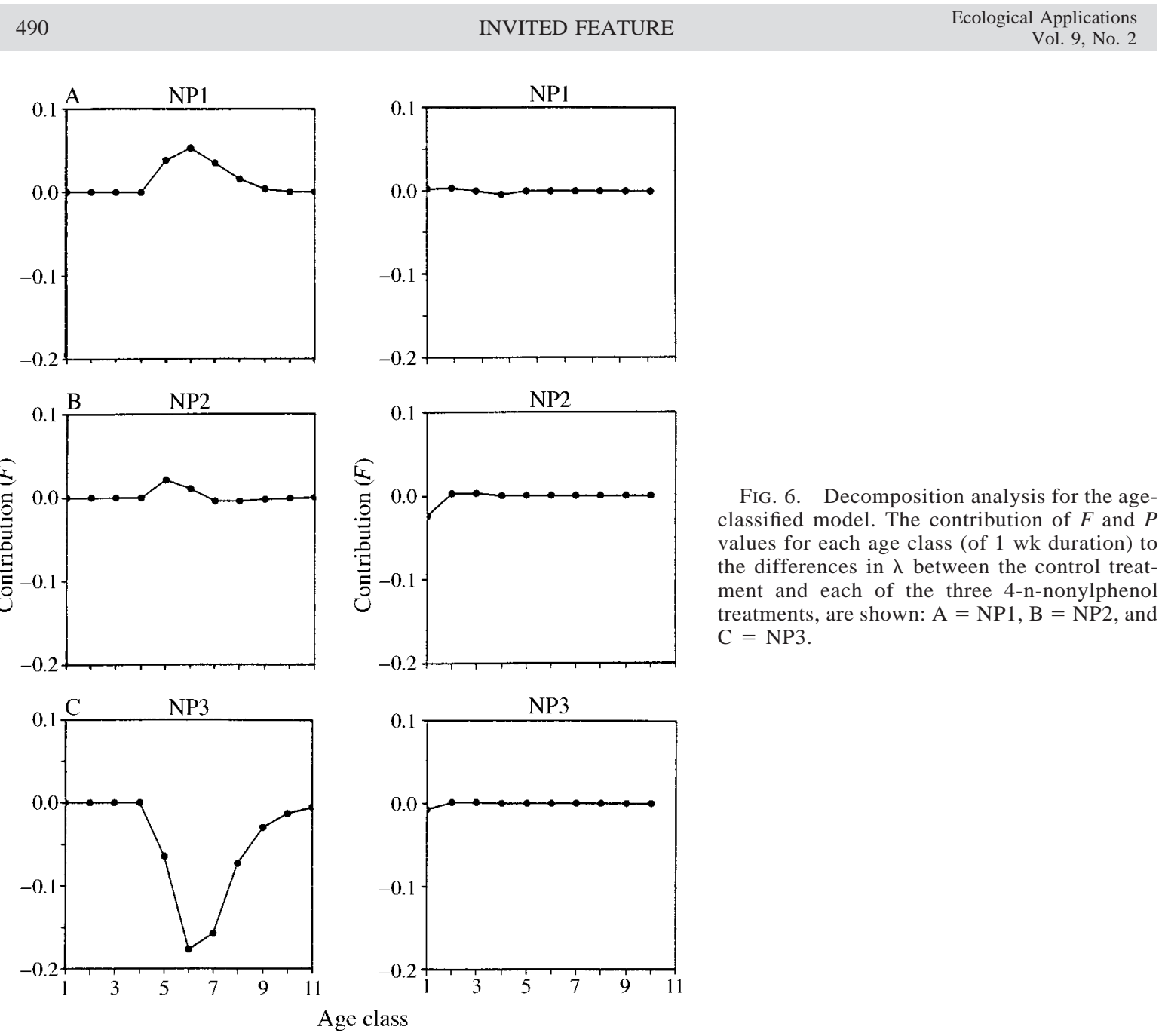

Decomposition analysis

Decomposition analysis of the age-classified model (Fig. 6A-C) showed that effects on fecundity during the first $4 \mathrm{wk}$ of reproduction (weeks 5-9 of the experiment) accounted for nearly all differences observed between the control treatment and both NP1 and NP3. After 9 wk of age, fecundity contributions were very small for all treatments. The age-classified survivorship terms only provided very small contributions to effects on $\lambda$ in all treatments.

Decomposition of the two-stage model (Fig. 7A-C) indicated that the effect of NP on time to first reproduction was a major cause of changes in population growth rate. Although time to first reproduction increased by only $\sim 20 \%$ in the NP3-treatment relative to NP0, it was responsible for more than half $(55 \%)$ of the effect on $\lambda$. In contrast, per individual fecundity decreased by $75 \%$, but only explained $44 \%$ of the effect on $\lambda$.

\section{Elasticity analysis}

Elasticity analysis performed on the two-stage model (Fig. 8) showed that population growth rate obtained from isolated hermaphrodites was several times more sensitive to changes in juvenile survival probability $\left(\sigma_{1}\right)$ than to changes in fecundity $(F)$. Adult survival probability $\left(\sigma_{2}\right)$ was even less important, with elasticity levels below those of fecundity. $\lambda$ appeared to be just as sensitive to changes in time to first reproduction $\left(t_{\mathrm{j}}\right)$ as to changes in juvenile survival $\left(\sigma_{\mathrm{T}}\right)$. The elasticity analysis explains the relatively high contribution of $t_{\mathrm{j}}$ in NP3 to effects on $\lambda$.

There was little if any difference in elasticity patterns among NP0, NP1, and NP2 (Fig. 8). However, there was a clear decrease in the relative sensitivity of $\lambda$ to changes in $t_{\mathrm{j}}$ in the NP3 treatment, and the elasticity of $F$ was slightly reduced as well. Thus, $\lambda$ became less sensitive to changes in $F$ and $t_{\mathrm{j}}$, but not $\sigma_{1}$ and $\sigma_{2}$, with increasing NP exposure.

\section{DISCUSSION}

\section{Life-history traits of isolated hermaphrodites}

Most of the life-table data obtained from isolated hermaphrodites in the control treatment in the present study are very similar to data reported from earlier 


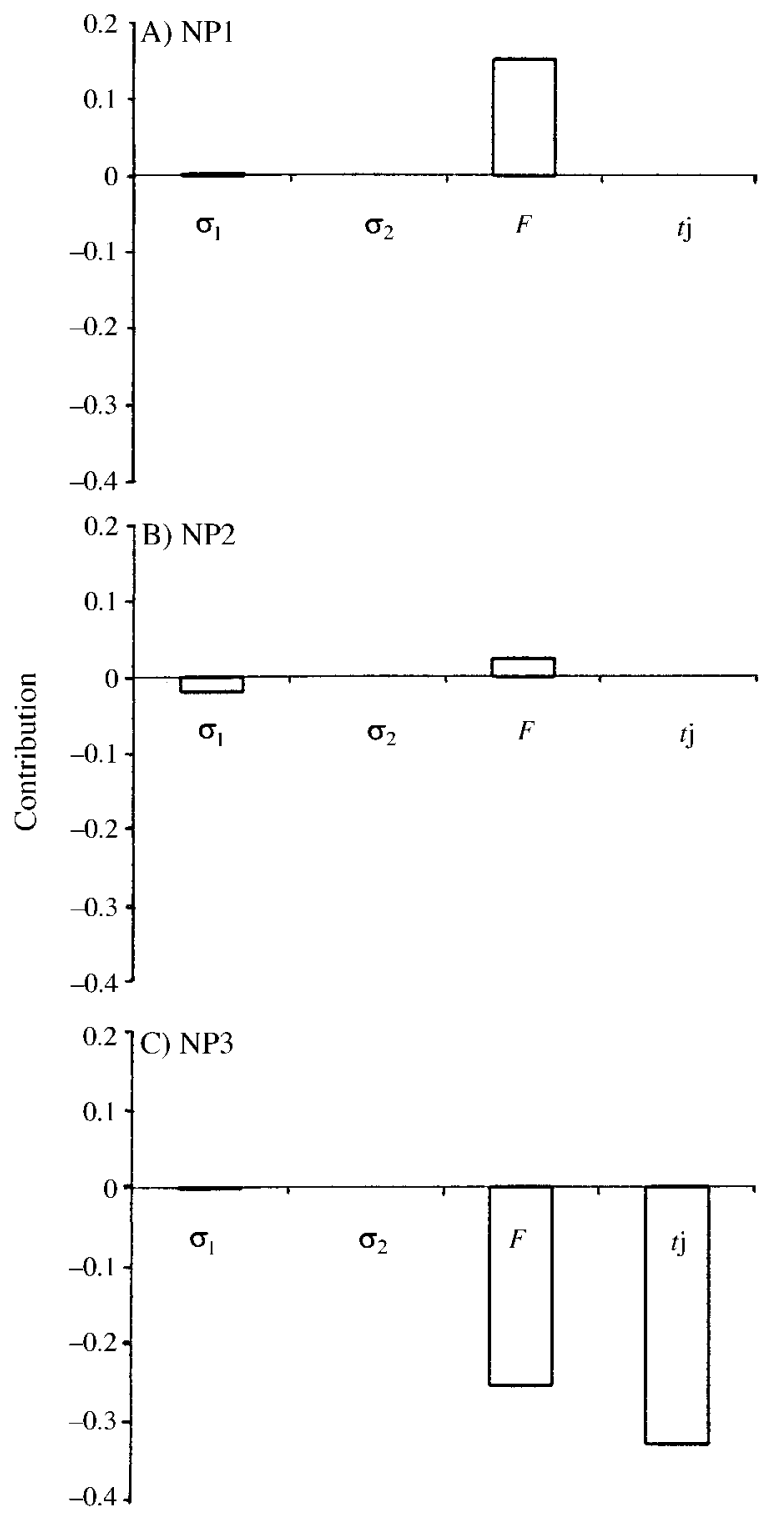

FIG. 7. Decomposition analysis for the two-stage model. The contribution of per-individual fecundity $(F)$, juvenile and adult survival probability per unit time $\left(\sigma_{1}\right.$ and $\left.\sigma_{2}\right)$, and time to first reproduction $\left(t_{\mathrm{j}}\right)$ to differences in $\lambda$ between the control treatment and each of the three 4-n-nonylphenol treatments: $\mathrm{A}=\mathrm{NP} 1, \mathrm{~B}=\mathrm{NP} 2$, and $\mathrm{C}=\mathrm{NP} 3$.

studies of Capitella sp. I (Eckelbarger et al. 1984, Tenore and Chesney 1985, Forbes and Lopez 1990, Bridges et al. 1994), suggesting that experimental conditions were favorable and that maintaining the experimental treatments in darkness had no measurable effects on the measured life-history traits.

Earlier work has shown considerable agreement in some of the life-history traits between hermaphrodites and females. Brood size, offspring survivorship, and number of eggs aborted per brood did not appear to differ between hermaphrodites and females (Petraitis

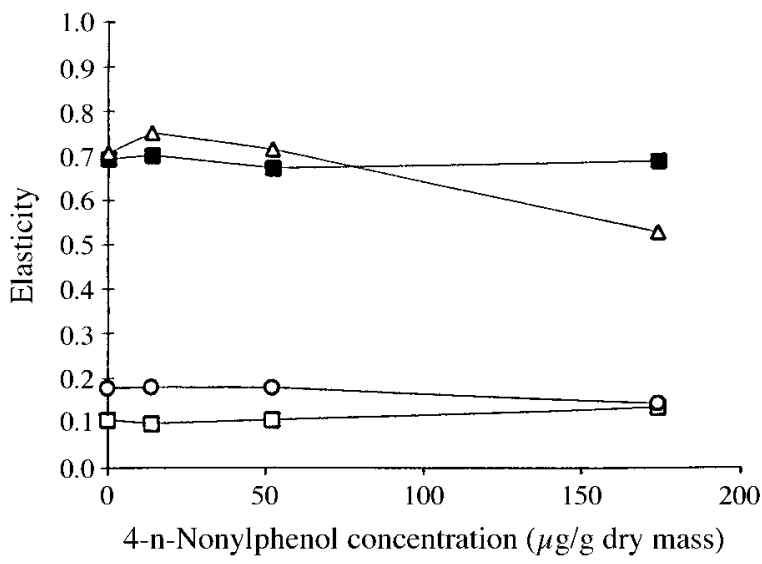

FIG. 8. Elasticities of per-individual fecundity $(F)$, juvenile and adult survival probability per unit time $\left(\sigma_{1}\right.$ and $\left.\sigma_{2}\right)$, and time to first reproduction $\left(t_{\mathrm{j}}\right)$ plotted against 4-nnonylphenol concentration: $\sigma_{1}=$ filled squares, $\sigma_{2}=$ empty squares, $F=$ circles, $t_{\mathrm{j}}=$ triangles.

1990). Breeding frequencies, however, were much lower among hermaphrodites reared in cultured cohorts (0.09-0.31 per day) compared with females (Petraitis 1988). Whether breeding frequency is density dependent is unreported, but our results show, on the contrary, that breeding frequencies obtained from isolated hermaphrodites are very similar to those reported for females at $20^{\circ} \mathrm{C}$ (once per 5-7 d) (Eckelbarger et al. 1984). Time to first reproduction might be slightly longer for hermaphrodites than the $30-40 \mathrm{~d}\left(20^{\circ} \mathrm{C}\right)$ reported for females (Grassle and Grassle 1976). This is supported by the fact that most of the morphological females identified on day 25 had oocytes visible in the coelom, whereas only a very small proportion of individuals with male characteristics showed the same properties. It is possible, however, that the presence of mature females on day 25 inhibited hermaphrodite development in males in the two lowest NP treatments and the control when most individuals had reached maturity.

\section{Effects of 4-n-nonylphenol}

The lowest tested concentration at which negative effects on Capitella sp. I were observed (i.e., the LOEC) in the present study was $174 \mu \mathrm{g} / \mathrm{g}$ dry mass giving a NOEC value of $52 \mu \mathrm{g} / \mathrm{g}$ dry mass. In comparison, the maximum acceptable toxicant concentration of nonylphenol reported for Chironomus tentans is $26.1 \mu \mathrm{g} / \mathrm{g}$ (Naylor 1995). The NOEC value in the present study is within a factor of 5 of the concentration range of nonylphenol reported in freshwater sediments (i.e., 0.24-13.1 $\mu \mathrm{g} / \mathrm{g}$, Ahel et al. 1990) and estuarine sediments (i.e., $0.15-13.7 \mu \mathrm{g} / \mathrm{g}$, Marcomini et al. 1990). Dividing our NOEC by a factor as small as 10 (which is probably lower than would be applied if this NOEC were to be used to calculate a predicted noeffect concentration (PNEC, Van Leeuwen and Her- 
mens 1995)) gives a value about half that of the highest measured environmental concentrations and suggests that this substance may pose a risk to aquatic systems.

In addition to the negative effects of NP detected at the highest test concentration, we observed stimulatory effects of this substance at the lowest test concentration. Stimulation at low toxicant concentrations, known as hormesis, has received increasing interest during the last two decades (e.g. Stebbing 1982, 1987, Sagan 1987, Calabrese and Baldwin 1993, Van Ewijk and Hoekstra 1993, Calabrese 1996). Chemically induced hormesis has been reported for a wide range of organisms including insects, worms, crabs, clams, oysters, fish, and various mammals, exposed to a large variety of agents (Stebbing 1982, Calabrese 1996). Positive effects on growth patterns are by far the most commonly reported type of hormesis, and since it has been observed for a variety of taxa and chemical agents, growth stimulation has been proposed as a general consequence of an overreaction of biosynthetic control systems (Stebbing 1987). Although determining effect concentrations for risk assessment generally considers only negative effects, positive effects on some species and not on others may affect community structure and should not be ignored.

Bridges et al. (1994) investigated the effects of different types of sediment organic content on survival, reproduction, and growth of Capitella sp. I. They found that worms started to reproduce before they reached asymptotic size for those treatments that permitted the largest body sizes and fastest growth rates. Treatments in which worms grew at the lowest rate and attained the smallest body size started to reproduce only after reaching the asymptotic body size. Our results show the opposite pattern. Worms started to reproduce before reaching asymptotic size in the highest NP treatment, whereas worms in NP0, NP1, and NP2 started to reproduce at about the time they reached asymptotic size. This could indicate that apart from reducing growth and reproduction, NP advances time to first reproduction, such that reproduction occurs at a smaller worm size than would have been expected otherwise.

\section{Demographic analysis}

The question as to whether population growth rate is more or less responsive to toxic stress than the most toxicant-responsive life-history trait is of great interest for predicting how results from toxicity tests of individual organism performance are related to effects at the population level. The significant stimulatory effect on brood size in the NP1 treatment did not affect population growth rate significantly, since $\lambda$ was insensitive to changes in fecundity. Thus, significant effects of NP on reproduction of individual Capitella sp. I were attenuated at the population level. With regard to negative effects, these occurred at the same test concentration for $\lambda$ as for time to maturity, time between broods, and fecundity. Therefore our results suggest that $\lambda$ is equally responsive (or less responsive if positive effects are included) than the individual life-history traits contributing to it.

Daniels and Allan (1981) found that population growth rates of Daphnia pulex exposed to copper were less responsive than juvenile survival, which was affected at a lower copper concentration. However, they questioned this conclusion, presumably because their study did not include any statistical evaluation of differences in population growth rates between treatments. Day and Kaushik (1987) showed that the intrinsic rate of increase ( $r$ ) of Daphnia galeata mendotae exposed to the insecticide fenvalerate was less responsive than number of offspring, which was affected at a lower dose. Likewise, Meyer et al. (1987) investigated Daphnia pulex's response to toxic levels of copper and cadmium, and found that population growth rate was not more responsive than any of the individual life-cycle parameters. Koivisto and Ketola (1995) reported similar results for Daphnia pulex exposed to copper in which significant effects on maturation time did not affect the population growth rate significantly. Another study by Pesch (1991) examined the effects of contaminated harbor sediment on the polychaete Neanthes arenaceodentata. Although significant effects on number of larvae per brood were observed, population growth rates were the same in all treatments. Bridges et al. (1994) investigated the effects of sediment amended with hydrocarbons on Capitella sp. I and showed significant differences in time to first reproduction. The demographic analysis of these data presented in Levin et al. (1996), however, showed no effect on population growth rate.

Overall, despite the limited number of investigations in which statistical comparisons were made, these studies suggest that, in terms of effect levels, population growth rate may be generally less responsive to toxicant stress than are the individual life-history traits contributing to it. However, it is essential to recognize that although effects on $\lambda$ may first become detectable at concentrations equal to or exceeding those causing effects on individual-level traits, a small percentage change in some traits may result in a much larger percentage change in $\lambda$. The elasticity analysis provides some information on how population growth rate will respond to changes in each of the individual life-history traits contributing to it, assuming that the traits are affected equally. Since effects are seldom equally distributed among traits, it is useful to apply decomposition analysis to determine how much the effects of a specific treatment on each life-history trait contribute to the changes observed in $\lambda$. For example, despite the high elasticity values for juvenile survival probability $\left(\sigma_{1}\right)$, the variation in $\sigma_{1}$ among treatments was not significant and did not contribute notably to the changes observed in $\lambda$. 
The importance of time to first reproduction for population growth rate has been emphasized by a number of demographic analyses (Caswell and Hastings 1980, Ebert 1985, Bridges et al. 1996, Kammenga et al. 1996, Levin et al. 1996). Kammenga et al. (1996) showed that small changes in time to first reproduction $\left(t_{\mathrm{j}}\right)$ in the nematode Plectus acuminatus exposed to cadmium had a large influence on population growth rate, even though $t_{\mathrm{j}}$ was not very responsive to cadmium. Levin et al. (1996) found that reduced age at maturity was the primary cause of Capitella sp. I's explosive response to sediment organic enrichment. The results of our two-stage model are similar in that a relatively small effect on $t_{\mathrm{j}}$ in the NP3 treatment resulted in the largest effect on $\lambda$, consistent with the high elasticity values for $t_{\mathrm{j}}$.

The elasticity analysis indicated that $\lambda$ was just as sensitive to changes in juvenile mortality as to changes in time to first reproduction, suggesting that recruitment failure (due to, e.g., competition or predation) would have a large impact on population growth rate. This may explain why opportunists such as Capitella sp. I do not dominate undisturbed and well-established communities as has been reported by several authors (Grassle and Grassle 1974, Gray 1979). On the other hand, $\lambda$ was much less sensitive to changes in per individual fecundity. This explains why no significant, positive effects were observed on $\lambda$ in the NP1 treatment despite significant stimulatory effects on both brood size and asymptotic body volume. This implies that even considerable effects on fecundity will not have a major effect on population growth rate in Capitella sp. I as long as food availability is high, as in the present experiment.

The elasticity patterns varied to some degree in response to NP exposure. When $F$ and $t_{\mathrm{j}}$ were affected negatively as a result of exposure to NP, the elasticity of $\lambda$ to changes in time to first reproduction $\left(t_{\mathrm{j}}\right)$ was markedly reduced and that to changes in $F$ slightly reduced. Thus, with increasing negative effects on $F$ and $t_{\mathrm{j}}, \lambda$ became less sensitive to changes in $F$ and especially $t_{\mathrm{j}}$, relative to adult and juvenile survival. Negative effects on fecundity terms, furthermore, increased the relative importance of adult survival. These results indicate that the relative impact on $\lambda$ decreased with increasing NP-exposure, e.g., the same effect on $t_{\mathrm{j}}$ in each of the NP-treatments and the control, had a decreasing impact on population growth rate with increasing NP exposure.

Most life-table response experiments, including the present study, are conducted under conditions in which density-dependent influences are minimal (i.e., population growth rates are positive). In nature, it is more likely that populations are at or near steady state, and density-dependent influences are likely to be of key importance for population dynamics (Calow et al. 1997, Sibly 1999). Furthermore, the consequences of toxicant impacts on $\lambda$ will vary depending on the starting value of $\lambda$, with $\lambda=1$ (i.e., the steady-state condition) being a critical threshold (Calow et al. 1997).

In conclusion, analysis of the effects of toxicant exposure on life-history traits via the application of demographic models can provide a powerful approach for determining the mechanistic basis of pollutant effects on population dynamics. Calculation of confidence limits and statistical tests of demographic parameters can greatly strengthen the interpretation of results from demographic models. The importance of density dependence and the initial state of population growth rate for determining population-level impacts of toxicants are key areas in need of study.

\section{ACKNOWLEDGMENTS}

We are grateful to A. G. Sørensen and B. Mørkøre for laboratory assistance. T. Lund provided valuable advice on the analytical chemical procedures. M. Niss and V. Andreassen shared their mathematical expertise. C. Agger and B. Larsen provided help with the statistical analyses. We thank R. Sibly and P. Calow for stimulating discussions on lifehistory theory, and T. Bridges, P. D. Christiansen, J. Kammenga, $\mathrm{H}$. Selck, and one anonymous reviewer for valuable comments on the manuscript.

\section{Literature Cited}

Ahel, M., W. Giger, and C. Schaffner. 1990. Behaviour of detergent derived organic chemicals in the Glatt river. 1. Alkylphenol polyethoxylates and their metabolites. Pages 470-475 in G. Angeletti and A. Bjørseth, editors. Organic micropollutants in the aquatic environment. Proceedings of the Sixth European Symposium held in Lisbon, Portugal, 22-24 May 1990. Kluwer Academic, Dordrecht, The Netherlands.

Bridges, T. S., R. Burres Wright, B. R. Gray, A. B. Gibson, and T. M. Dillon. 1996. Chronic toxicity of Great Lakes sediments to Daphnia magna: elutriate effects on survival, reproduction and population growth. Ecotoxicology 5:83102.

Bridges, T. S., L. A. Levin, D. Cabrera, and G. Plaia. 1994. Effects of sediment amended with sewage, algae, or hydrocarbons on growth and reproduction in two opportunistic polychaetes. Journal of Experimental Marine Biology and Ecology 177:99-119.

Calabrese, E. J. 1996. Expanding the RfD concept to incorporate and optimize beneficial effects while preventing toxic responses from nonessential toxicants. Ecotoxicology and Environmental Safety 34:94-101.

Calabrese, E. J., and L. A. Baldwin. 1993. Possible examples of chemical hormesis in a previously published study. Journal of Applied Toxicology 13:169-172.

Calow, P., and V. E. Forbes. 1999. Manual on risk assessment of subregional sea areas: a practical guide for tropical ecosystems. GEF/UNDP/IMO Regional Programme for the Prevention and Management of Marine Pollution in the East Asian Seas, Manila, The Philippines, in press.

Calow, P., R. M. Sibly, and V. E. Forbes. 1997. Risk assessment on the basis of simplified population dynamics' scenarios. Environmental Toxicology and Chemistry 16: 1983-1989.

Caswell, H. 1989a. Matrix population models-construction, analysis, and interpretation. Sinauer Associates, Inc. Publishers, Sunderland, Massachusetts, USA.

. 1989b. Analysis of life table response experiments I. Decomposition of effects on population growth rate. Ecological Modelling 46:221-237. 
1996. Analysis of life table response experiments II. Alternative parameterizations for size- and stage-structured models. Ecological Modelling 88:73-82.

Caswell, H., and A. Hastings. 1980. Fecundity, developmental time, and population growth rate: an analytical solution. Theoretical Population Biology 17:71-79.

Caswell, H., R. Naiman, and R. Morin. 1984. Evaluation of the consequences of reproduction in complex salmonid life cycles. Aquaculture 43:123-143.

Comber, M. H. I., T. D. Williams, and K. M. Stewart. 1993. The effects of nonylphenol on Daphnia magna. Water Research 27:273-276.

Daniels, R. E., and J. D. Allan. 1981. Life table evaluation of chronic exposure to a pesticide. Canadian Journal of Fisheries and Aquatic Sciences 38:485-494.

Day, K., and N. K. Kaushik. 1987. An assessment of the chronic toxicity of the synthetic pyrethroid, fenvalerate, to Daphnia galeata mendotae, using life tables. Environmental Pollution 44:13-26.

De Kroon, H., A. Plaisier, J. Van Groenendael, and H. Caswell. 1986. Elasticity: the relative contribution of demographic parameters to population growth rate. Ecology 67: 1427-1431

Ebert, T. A. 1985. Sensitivity of fitness to macroparameter changes: an analysis of survivorship and individual growth in sea urchin life histories. Oecologia 65:461-467.

Eckelbarger, K. J., P. A. Linley, and J. P. Grassle. 1984. Role of ovarian follicle cells in vitellogenesis and oocyte resorption in Capitella sp. I (Polychaeta). Marine Biology 79:133-144.

Forbes, T. L., V. E. Forbes, and M. H. Depledge. 1994. Individual physiological responses to environmental hypoxia and organic enrichment: implications for early soft-bottom community succession. Journal of Marine Research 52: 1081-1100.

Forbes, T. L., and G. R. Lopez. 1987. The allometry of deposit feeding in Capitella species I (Polychaeta: Capitellidae): the role of temperature and pellet weight in the control of egestion. Biological Bulletin 172:187-201.

Forbes, T. L., and G. R. Lopez. 1990. Ontogenetic changes in individual growth and egestion rates in the deposit-feeding polychaete Capitella sp. I. Journal of Experimental Marine Biology and Ecology 143:209-220.

Giger, W., P. H. Brunner, and C. Schaffner. 1984. 4-Nonylphenol in sewage sludge: accumulation of toxic metabolites from nonionic surfactants. Science 225:623-625.

Giger, W., E. Stephanou, and C. Schaffner. 1981. Persistent organic chemicals in sewage effluents: 1. Identification of nonylphenolethoxylates by glass capillary gas chromatography/mass spectrometry. Chemosphere 10:1253-1263.

Granmo, Å., R. Ekelund, K. Magnusson, and M. Berggren. 1989. Lethal and sublethal toxicity of 4-nonylphenol to the common mussel (Mytilus edulis L.). Environmental Pollution 59:115-127.

Grassle, J. F., and J. P. Grassle. 1974. Opportunistic life histories and genetic systems in marine benthic polychaetes. Journal of Marine Research 32:253-284.

Grassle, J. P. 1980. Polychaete sibling species. Pages 25-31 in R. O. Brinkhurst and D. G. Cook, editors. Aquatic oligochaete biology. Plenum Publishing, New York, New York, USA.

Grassle, J. P., and J. F. Grassle. 1976. Sibling species in the marine pollution indicator Capitella (Polychaeta). Science 192:567-569.

Gray, J. S. 1979. Pollution-induced changes in populations. Philosophical Transactions of the Royal Society of London B286:545-561.

Hedges, J. I., and J. H. Stern. 1984. Carbon and nitrogen determinations of carbonate-containing solids. Limnology and Oceanography 29:657-663.
Holbrook, M. J. L., and J. P. Grassle. 1984. The effect of low density on the development of simultaneous hermaphroditism in male Capitella sp. I (Polychaeta). Biological Bulletin 166:103-109.

Jensen, S., L. Renberg, and L. Reutergård. 1977. Residue analysis of sediment and sewage sludge for organochlorines in the presence of elemental sulfur. Analytical Chemistry 49:316-318.

Kammenga, J. E., M. Busschers, N. M. Van Straalen, P. C. Jepson, and J. Bakker. 1996. Stress induced fitness reduction is not determined by the most sensitive life-cycle trait. Functional Ecology 10:106-111.

Kaufmann, K. W. 1981. Fitting and using growth curves. Oceologia 49:293-299.

Koivisto, S., and M. Ketola. 1995. Effects of copper on lifehistory traits of Daphnia pulex and Bosmina longirostris. Aquatic Toxicology 32:255-269.

Leslie, P. H. 1945. On the use of matrices in certain population mathematics. Biometrika 33:183-212.

Levin, L., H. Caswell, T. Bridges, C. Dibacco, D. Cabrera, and G. Plaia. 1996. Demographic responses of estuarine polychaetes to sewage, algal, and hydrocarbon additions: life-table response experiments. Ecological Applications 6: 1295-1313.

Marcomini, A., B. Pavoni, A. Sfriso, and A. A. Orio. 1990. Persistent metabolites of alkylphenol polyethoxylates in the marine environment. Marine Chemistry 29:307-323.

McLeese, D. W., V. Zitko, C. D. Metcalfe, and D. B. Sergeant. 1980. Lethality of aminocarb and the components of the aminocarb formulation to juvenile atlantic salmon, marine invertebrates and a freshwater clam. Chemosphere 9:7982.

McLeese, D. W., V. Zitko, D. B. Sergeant, L. Burridge, and C. D. Metcalfe. 1981. Lethality and accumulation of alkylphenols in aquatic fauna. Chemosphere 10:723-730.

Meyer, J. S., C. G. Ingersoll, and L. L. McDonald. 1987. Sensitivity analysis of population growth rates estimated from cladoceran chronic toxicity tests. Environmental Toxicology and Chemistry 6:115-126.

Meyer, J. S., C. G. Ingersoll, L. L. McDonald, and M. S. Boyce. 1986. Estimating uncertainty in population growth rates: jackknife vs. bootstrap techniques. Ecology 67:11561166.

Naylor, C. G. 1995. Environmental fate and safety of nonylphenol ethoxylates. Textile Chemist and Colorist 27(4): 29-33.

Nimrod, A. C., and W. H. Benson. 1996. Environmental estrogenic effects of alkylphenol ethoxylates. Critical Rewiews in Toxicology 26:335-364.

Pechenik, J. A., and T. R. Cerulli. 1991. Influence of delayed metamorphosis on survival, growth and reproduction of the marine polychaete Capitella sp. I. Journal of Experimental Marine Biology and Ecology 151:17-27.

Pesch, C. E. 1991. Effects of a contaminated sediment on life-history traits and population growth rate of Neanthes arenaceodentata (Polychaeta: Nereidae) in the laboratory. Environmental Toxicology and Chemistry 10:805-815.

Petraitis, P. S. 1985. Females inhibit males' propensity to develop into simultaneous hermaphrodites in Capitella species I (Polychaeta). Biological Bulletin (Woods Hole) 168: 395-402.

. 1988. Occurrence and reproductive success of feminized males in the polychaete Capitella capitata (Species Type I). Marine Biology 97:403-412.

. 1990. Dynamics of sex change in a capitellid polychaete. Lectures on Mathematics in the Life Sciences 22: 127-154.

Sagan, L. A. 1987. What is hormesis and why haven't we heard about it before? Health Physics 52:521-525.

Sanders, H. L., J. F. Grassle, G. R. Hampson, L. S. Morse, 
S. Garner-Price, and C. C. Jones. 1980. Anatomy of an oil spill: Long-term effects from the grounding of the barge Florida off West Falmouth, Massachusetts. Journal of Marine Research 38:265-380.

Sibly, R. M. 1999. Efficient experimental designs for studying stress and population density in animal populations. Ecological Applications 9:496-503.

Siegel, S., and N. J. Castellan, Jr. 1988. Nonparametric statistics for behavioral sciences. McGraw-Hill, New York, New York, USA.

Stebbing, A. R. D. 1982. Hormesis-the stimulation of growth by low levels of inhibitors. Science of the Total Environment 22:213-234.

1987. Growth hormesis: a by-product of control. Health Physics 52:543-547.

Sweetman, A. J. 1993. Development and application of a multi-residue analytical method for the determination of n-alkanes, linear alkylbenzenes, polynuclear aromatic hydrocarbons and 4-nonylphenol in digested sewage sludge. Water Research 28:343-353.

Tenore, K. R., and E. J. Chesney. 1985. The effects of interaction of rate of food supply and population density on the bioenergetics of the opportunistic polychaete, Capitella capitata (type I). Limnology and Oceanography 30:11881195.

Van Ewijk, P. H., and J. A. Hoekstra. 1993. Calculation of the EC50 and its confidence interval when stimulus is present. Ecotoxicology and Environmental Safety 25:25-32.

Van Leeuwen, C. J., and J. L. M. Hermens. 1995. Risk assessment of chemicals. Kluwer Academic, Dordrecht, The Netherlands.

Wahlberg, C., L. Renberg, and U. Wideqvist. 1990. Determination of nonylphenol ethoxylates as their pentafluorobenzoates in water, sewage sludge and biota. Chemosphere 20:179-195.

Ward, T. J., and P. A. Hutchings. 1996. Effects of trace metals on infaunal species composition in polluted intertidal and subtidal marine sediments near a lead smelter, Spencer Gulf, South Australia. Marine Ecology Progress Series 135: 123-135.

Weinberger, P., C. DeChacin, and M. Czuba. 1987. Effects of nonyl phenol, a pesticide surfactant, on some metabolic processes of Chlamydomonas segnis. Canadian Journal of Botany 65:696-702.

Zar, J. H. 1974. Biostatistical analysis. Prentice-Hall, Englewood Cliffs, New Jersey, USA. 